\title{
Proteomic analysis of synovial fluid in osteoarthritis using SWATH-mass spectrometry
}

\author{
WEIXIONG LIAO ${ }^{1}$, ZHONGLI LI ${ }^{2}$, TANSHI LI ${ }^{1}$, QIANG ZHANG ${ }^{2}$, HENG ZHANG $^{1}$ and XINZHENG WANG ${ }^{3}$ \\ Departments of ${ }^{1}$ Emergency and ${ }^{2}$ Orthopedics, Chinese PLA General Hospital, Beijing 100853; \\ ${ }^{3}$ National Center of Biomedical Analysis, Beijing 100850, P.R. China
}

Received October 27, 2016; Accepted June 16, 2017

DOI: $10.3892 / \mathrm{mmr} .2017 .8250$

\begin{abstract}
The lack of early diagnostic maneuvers and effective pharmacotherapy for osteoarthritis (OA) is predominantly attributed to current limited understanding of its pathogenesis. In the present study, the alteration of synovial fluid (SF) proteome in OA were analyzed using SWATH-mass spectrometry (SWATH-MS) to further elucidate the pathogenesis of OA. SF samples were collected from $10 \mathrm{OA}$ and 10 rheumatoid arthritis (RA) patients undergoing arthroplasty and 10 patients undergoing arthroscopy for traumatic arthritis (meniscus injury without cartilage lesion). According to the Kellgren-Lawrence (KL) radiographic grading criteria, all the OA and RA patients were classified as KL grade 4. SWATH-MS was applied to identify differentially expressed proteins specifically regulated in OA. Differentially expressed proteins identified by SWATH-MS were subjected to gene ontology (GO) and Kyoto Encyclopedia of Genes and Genomes (KEGG) pathway annotation. Proteins of interest were quantified by enzyme-linked immunosorbent assay (ELISA) following identification. With the use of SWATH-MS, 131 proteins were identified to be differentially expressed in OA, of which 93 corresponded to upregulation and 38 to downregulation. Complement C1r was the most significantly upregulated protein in OA. Twenty-eight out of the 131 proteins were specifically regulated in OA, of
\end{abstract}

Correspondence to: Professor Zhongli Li, Department of Orthopedics, Chinese PLA General Hospital, 28 Fuxing Road, Beijing 100853, P.R. China

E-mail: lizhongli301@163.com

Abbreviations: DDA, data-dependent acquisition; DDR-2, discoidin domain receptor 2; DKK2, Dickkopf-related protein 2; ECM, extracellular matrix; ELISA, enzyme-linked immunosorbent assay; GO, gene ontology; GST, glutathione S-transferase; ICAM-1, intercellular adhesion molecule-1; KEGG, Kyoto Encyclopedia of Genes and Genomes; LP, link protein; MAC, membrane attack complex; OA, osteoarthritis; RA, rheumatoid arthritis; SF, synovial fluid; SWATH-MS, SWATH-mass spectrometry; TIMP-1, tissue inhibitor of metalloproteinase-1

Key words: proteomics, synovial fluid, osteoarthritis, SWATH-mass spectrometry, complement C1r, dickkopf-related protein 2 which 17 were upregulated and 11 were downregulated. Dickkopf-related protein 2 (DKK2) was one of the proteins specifically upregulated in OA. Furthermore, KEGG pathway annotation indicated that differentially expressed proteins in OA were enriched in complement and coagulation cascades. ELISA indicated that OA severity was positively correlated with the levels of complement $\mathrm{C} 1 \mathrm{r}(\mathrm{r}=0.549 ; \mathrm{P}<0.001)$ and DKK2 $(r=0.79 ; \mathrm{P}<0.001)$ in the $\mathrm{SF}$. The results indicate that complement and coagulation cascades are involved in the pathogenesis of OA. Differentially expressed proteins, including complement $\mathrm{C} 1 \mathrm{r}$ and DKK2 may be used as potential biomarkers or drug targets, which may facilitate with intervention of $\mathrm{OA}$.

\section{Introduction}

Osteoarthritis (OA) is a degenerative joint disorder characterized by articular cartilage degradation, osteophyte formation, synovium hyperplasia, subchondral bone cysts and sclerosis. It is one of the leading causes of pain and dysfunction in the joints among the aging population (1). Despite the high prevalence of OA, its pathogenesis remains unclear. At present, the diagnosis of OA primarily depends on clinical symptoms and radiographic findings. However, the majority of individuals are clinically asymptomatic at the early stages of OA, and pathological degradation of the articular cartilage already exists before the symptoms arise. Furthermore, radiographs may appear normal at this stage due to lack of sensitivity in visualizing minimal cartilage lesions. Thus, the diagnostic methods currently in use have limitations in detecting preclinical OA and provide an inaccurate assessment of disease progression. Although multiple translational techniques detect early OA processes, it remains unknown as to whether they truly represent the initial events. Therefore, the clinical diagnosis of early $\mathrm{OA}$ is one of the greatest challenges in this field (2). Currently, there is no definitely effective medication for the treatment of early OA. Traditional medications, such as glucosamine and hyaluronic acid, once thought of as effective, have no longer been recommended by the latest American Academy of Orthopaedic Surgeons guideline due to evidence of a lack of efficacy (3). The medications currently used for the treatment of OA are predominantly aimed at relieving pain, but do not prevent disease progression. These limitations give rise to the interest in proteomic studies to identify candidate biomarkers 
and drug targets, which may facilitate early diagnosis and intervention of OA. Additionally, proteomic studies provide greater insight into $\mathrm{OA}$ pathogenesis and original insights into the molecular mechanisms involved in disease progression.

Advances in proteomic technologies have facilitated extensive proteomic characterization of various body fluids, including serum (4) and urine (5) in OA. However, evaluating the synovial fluid (SF) proteome is more likely to yield a higher concentration of potential biomarkers than serum and urine, as SF is in direct contact with articular cartilage, synovium, ligament, meniscus and joint capsule. Alterations in metabolism of any of these tissues during disease progression are reflected as alterations in the proteomic profile of SF, which is also less influenced by systematic conditions. Therefore, it is considered more appropriate to detect the proteomic profile in SF than in other body fluids. There are also certain studies designed to investigate the expression of certain proteins in SF from OA patients (6). These studies focused on evaluating the expression of certain proteins, but failed to screen novel proteins that are differentially expressed in SF. With the use of quantitative proteomic technologies, a series of novel proteins differentially expressed in SF from OA patients have been identified in previous studies $(7,8)$. However, the majority of these investigations were performed using low resolution mass spectrometry (MS) and with minimal fractionation of the samples, which limited the depth of coverage. Isobaric tags for relative and absolute quantification is a labeling method with high throughput, easy automation, high resolution and quantification accuracy, which enables quantitative proteomics analysis of four samples simultaneously under the same experimental conditions (9). This technology has also been applied to investigate alterations of the proteomic profile in OA in previous studies $(10,11)$. Although a greater number of novel proteins were identified to be differentially expressed using this method $(10,11)$, whether these proteins are specifically regulated in OA remains unclear due to a lack of effective standard controls or inter-controls.

SWATH-MS is an emerging data-independent acquisition method, whichgenerates a single file containing fragment ion spectra of all ionized species of a sample (12). When combined with a targeted data analysis strategy, SWATH-MS was demonstrated to achieve favorable accuracy, dynamic range, and reproducibility of selected reaction monitoring, which is considered to be the golden standard in quantitative proteomic technology (13). In order to identify differentially expressed proteins specifically regulated in OA and further elucidate the disease pathogenesis, SWATH-MS was performed to compare the SF proteome in OA, rheumatoid arthritis (RA) and traumatic arthritis (meniscus injury without cartilage defect). Subsequently, proteins of interest were quantified by enzyme-linked immunosorbent assay (ELISA). To the best of our knowledge, no comparative analysis with respect to the SF proteome has been conducted in these three types of knee disorders using SWATH-MS.

\section{Materials and methods}

Subjects. The present study was initiated following approval from the Ethical Committee of the Chinese PLA General Hospital and informed consent was obtained from all the subjects. SF samples were acquired from 30 patients who had received surgical treatment for a knee disorder between September 2014 and January 2015 in the Orthopedics Department of the Chinese PLA General Hospital (Beijing, China). A total of 10 knee OA patients (4 men and 6 women; age, 64-72 years; mean age, 67 years) undergoing arthroplasty were defined as the study group, 10 knee RA patients (4 men and 6 women; age, 45-69 years; mean age, 60 years) undergoing arthroplasty served as the inter-control group, and 10 patients ( 7 men and 3 women; age, 23-47 years; mean age, 29 years) undergoing arthroscopy for traumatic knee arthritis (meniscus injury without cartilage defect) within 7 days after injury served as the standard control group. The diagnosis of knee OA and RA was based upon the criteria of the American College of Rheumatology (14). The severity of knee OA and RA was evaluated by the Kellgren-Lawrence (KL) radiographic grading criteria (Fig. 1), according to which all the knee OA and RA patients recruited were classified as KL grade 4. All the patient data were retrieved from our institutional database through chart review.

Sample preparation and protein digestion. SF samples (1-2 ml) were aspirated from the affected knee using a sterile puncture needle prior to surgery and $50 \mu \mathrm{l}$ proteinase inhibitor cocktail (Roche Molecular Diagnostics, Pleasanton, CA, USA) was immediately added to the sample after collection. The mixture was centrifuged at $19,228 \mathrm{x} \mathrm{g}$ for $20 \mathrm{~min}$ at $4^{\circ} \mathrm{C}$ (Hettich MIKRO 22R; Hettich Benelux, Tuttlingen, Germany) to remove cells and debris. For the comprehensive shotgun analysis, all the SF samples were depleted of the 14 most abundant proteins with the multiple affinity removal system (MARS Hu-14 spin cartridge; Agilent Technologies, Inc., Santa Clara, CA, USA) according to the manufacturer's instruction. After reduction and alkylation with $10 \mathrm{mM}$ tris (2-carboxyethyl) phosphine (Sigma-Aldrich; Merck KGaA, Darmstadt, Germany) and $20 \mathrm{mM}$ iodoacetamide (Sigma-Aldrich; Merck $\mathrm{KGaA}$ ), the samples were subjected to protein quantification using the Bradford Assay according to the manufacturer's instructions with a commercial Bradford reagent (Bio-Rad Laboratories, Hercules, CA, USA). Subsequently, the samples were diluted with $1 \mathrm{M}$ urea and digested with sequencing-grade trypsin (Promega Corporation, Madison, WI, USA) at a protease/protein ratio of $1: 30$ overnight at $37^{\circ} \mathrm{C}$. Following digestion, the samples were stored at $-80^{\circ} \mathrm{C}$ until use.

Shotgun proteomic measurement. The processed peptides were measured using a Triple TOF 5600 Plus mass spectrometer (SCIEX, Framingham, MA, USA) operated in data-dependent acquisition (DDA) mode. The mass spectrometer was interfaced with a NanoLC Ultra 2D Plus HPLC system (Eksigent Technologies, Dublin, CA, USA) as previously described $(12,13)$. Peptides were directly injected onto a $20-\mathrm{cm}$ PicoFrit emitter and separated using a 120-min gradient from 2-35\% (buffer A: $0.1 \%$ Formic acid and $2 \%$ acetonitrile; buffer B: $0.1 \%$ Formic acid and $90 \%$ acetonitrile) at a flow rate of $300 \mathrm{nl} / \mathrm{min}$. MS1 spectra were collected in the range $350-1,250 \mathrm{~m} / \mathrm{z}$ for $250 \mathrm{msec}$. The 20 most intense precursors with charge state 2-5, which exceeded 250 counts per sec were selected for fragmentation, and MS2 spectra were collected in the range $100-1,500 \mathrm{~m} / \mathrm{z}$ for $50 \mathrm{msec}$. The precursor ions were dynamically excluded from reselection for $20 \mathrm{sec}$. 

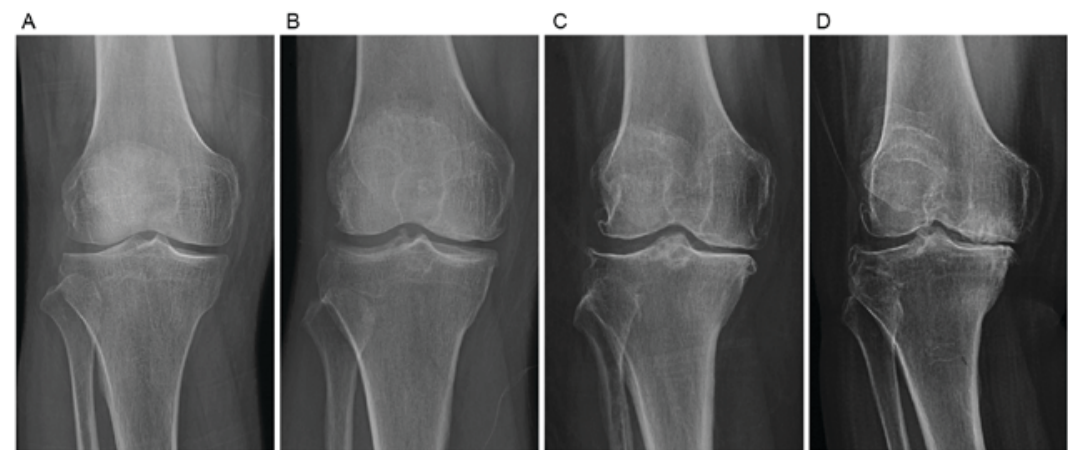

Figure 1. The Kellgren-Lawrence radiographic grading criteria. (A) Grade 1, doubtful narrowing of joint space and possible osteophytic lipping. (B) Grade 2 , definite osteophytes and possible narrowing of joint space. (C) Grade 3, moderate multiple osteophytes, definite narrowing of joints space, some sclerosis and possible deformity of bone contour. (D) Grade 4, large osteophytes, marked narrowing of joint space, severe sclerosis and definite deformity of bone contour.

SWATH-MS measurement. SWATH-MS measurements were performed with peptide mixtures generated by digesting SF samples. The unfractionated, total peptide samples were analyzed to minimize confounding factors introduced by sample handling. The same liquid chromatography-MS/MS system used for DDA measurements was used for SWATH analysis $(12,13)$. In SWATH-MS mode, the instrument was specifically tuned to optimize the quadrupole settings for the selection of $25-\mathrm{m} / \mathrm{z}$ wide precursor ion selection windows. Using an isolation width of $25 \mathrm{~m} / \mathrm{z}$, a set of overlapping windows was constructed, covering the precursor mass range of 400-1,200 m/z. The effective isolation windows were considered as being 400-425, 424-449, 448-473 m/z, and so on. SWATH-MS2 spectra were collected from 100-1,500 m/z. The collision energy was optimized for each window according to the calculation for a charge $2^{+}$ion centered upon the window with a spread of $15 \mathrm{eV}$. An accumulation time of $50 \mathrm{msec}$ was used for all fragment ion scans in high-sensitivity mode, and for each SWATH-MS cycle, a survey scan in high-resolution mode was acquired for 50 msec. SWATH-MS measurements were repeated three times on each sample to access the technical variability. Differentially expressed proteins identified by SWATH-MS were subjected to gene ontology (GO) and KEGG pathway annotation.

ELISA. Another $90 \mathrm{SF}$ samples were obtained from $36 \mathrm{knee} \mathrm{OA}$ patients (17 men and 19 women, aged 57-74 years; mean age, 65 years), 36 knee RA patients (15 men and 21 women, aged 41-70 years; mean age, 58 years) and 18 patients (12 men and 6 women, aged 21-47 years;mean age, 28 years) with traumatic knee arthritis (meniscus injury without cartilage defect) to detect the level of complement Clr and that of Dickkopf-related protein 2 (DKK2). According to the KL radiographic grading criteria, 18 out of the 36 knee OA patients were classified as KL grade 2, and the other 18 as KL grade 4. Similarly, 18 out of the 36 knee RA patients were categorized as KL grade 2, and the other 18 as KL grade 4 . The levelsof complement $\mathrm{C} 1 \mathrm{r}$ and that of DKK2 in SF were measured by ELISA (MyBioSource; R\&D Systems, Inc., Minneapolis, MN, USA) according to the manufacturer's instructions.

Statistical analysis. Statistical analysis was performed with SPSS software (Version 17.0; SPSS Inc, Chicago, IL, USA). Data were expressed as the mean \pm standard deviation. One way analysis of variance followed by a post hoc Tukey test for multiple comparisons was performed to compare the quantitative data from different groups. Pearson's correlation coefficient was used to analyze the correlation between protein level and disease severity. $\mathrm{P}<0.05$ was considered to indicate a statistically significant difference.

\section{Results}

SWATH-MS. To evaluate technical variability, each sample was analyzed in triplicate. As presented in Fig. 2, total ion current chromatograms from triplicates of the same sample exhibited high similarity, whereas those from different samples displayed obvious variation. The results revealed small technical variability and excellent reproducibility of SWATH-MS measurements and great variation in proteomic profiles of SF from the different groups.

Proteins were regarded to be differentially expressed when ion intensity between groups was significantly different $(\mathrm{P}<0.05)$ and fold change between groups was $>1.5$. Based on these criteria, the comparison between OA and traumatic arthritis resulted in the identification of 131 significantly different proteins, of which 93 corresponded to upregulation and 38 to downregulation in OA. Complement $\mathrm{Clr}$ was the most significantly upregulated protein in OA (Fig. 3). The comparison between RA and traumatic arthritis led to the identification of 185 significantly different proteins, of which 152 corresponded to upregulation and 33 to downregulation in RA. The comparison between OA and RA gave rise to the identification of 139 significantly different proteins, of which 22 corresponded to upregulation and 117 to downregulation in OA.

Proteins with a fold change $>1.5$ in OA and a fold change $<1.5$ in RA were defined as differentially expressed proteins specifically regulated in OA. On the basis of these criteria, 28 proteins were considered to be specifically regulated in OA, of which 17 were upregulated and 11 were downregulated (Table I). DKK2 was one of the proteins specifically upregulated in OA (Fig. 4). Proteins up-/downregulated with a fold change $>1.5$ in OA and down-/upregulated with a fold change $>1.5$ in RA were defined as differentially expressed proteins conversely regulated in $\mathrm{OA}$ and $\mathrm{RA}$. According to these criteria, 8 proteins were identified to be conversely regulated in OA and RA, of which 3 were upregulated in OA and downregulated in RA, 5 were downregulated in OA and upregulated in RA (Table II). 


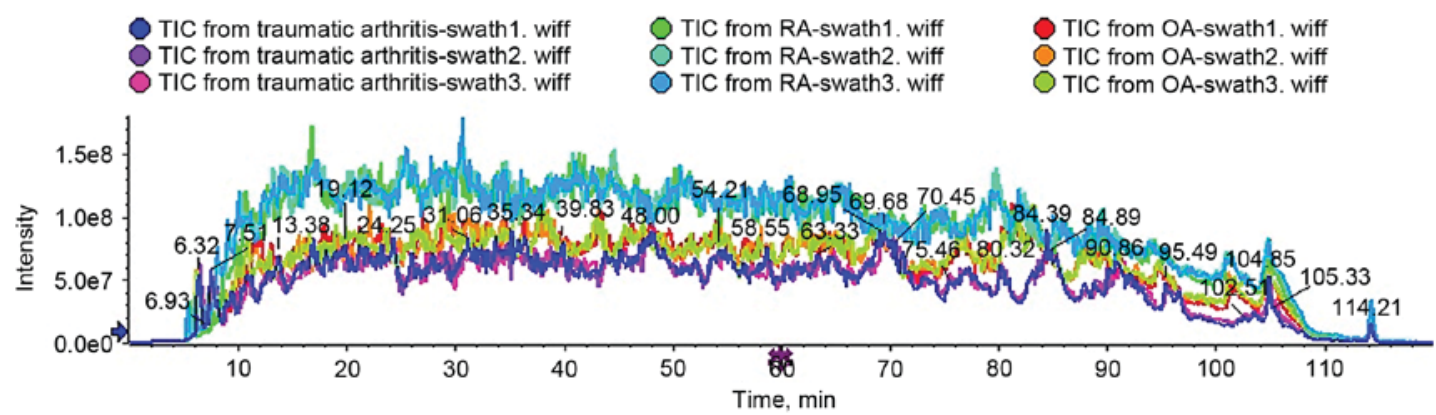

Figure 2. TIC chromatogram from traumatic arthritis, RA and OA using liquid chromatography-MS/MS analysis. The chromatogram demonstrated an effective chromatographic separation for the SF proteome. The peaks existed all the time rather than only fora period of time. The shapes of all chromatographic peaks were sharp and symmetrical. In addition, this chromatogram indicated small technical variability and excellent reproducibility of SWATH-MS and great variation in the SF proteome from the different groups. TIC, total ion current; RA, rheumatoid arthritis; OA, osteoarthritis; MS, mass spectrometry; SF, synovial fluid.
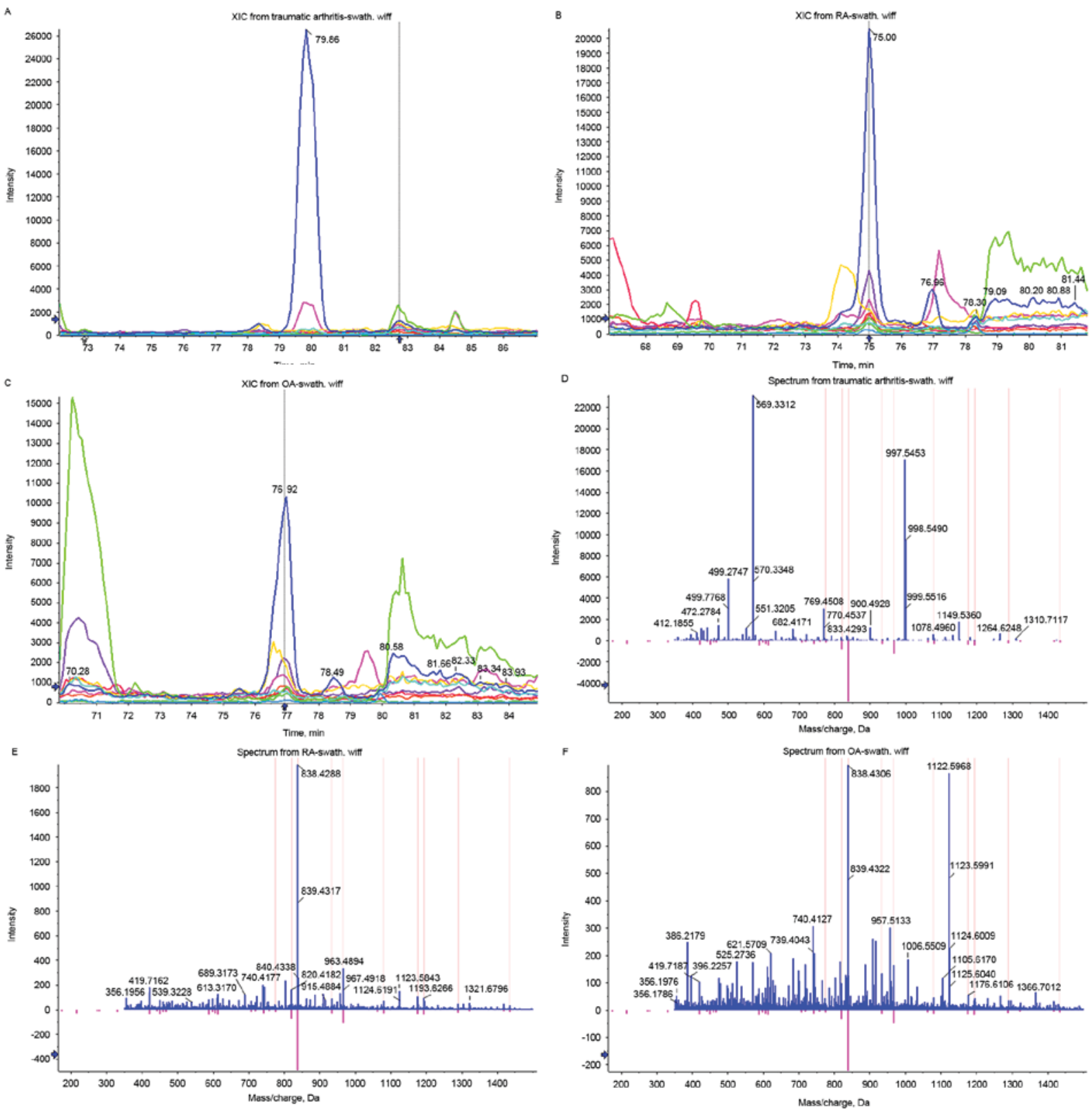

Figure 3. Quantification results of complement C1r using SWATH-MS.XICs demonstrated the relative abundance of peptides from complement C1r in SF from (A) traumatic arthritis, (B) RA and (C) OA. Spectrum of peptides from complement C1r in SF from (D) traumatic arthritis, (E) RA and (F) OA. Relative quantification results were derived from comparing the summed XIC peak areas of the fragment ion peak groups, as indicated by the arrow. XIC, extracted ion chromatograms; MS, mass spectrometry; SF, synovial fluid; RA, rheumatoid arthritis; OA, osteoarthritis. 
Table I. Differentially expressed proteins specifically regulated in osteoarthritis identified by SWATH-mass spectrometry.

\begin{tabular}{|c|c|c|c|c|c|}
\hline Protein ID & Accession & Protein name & t-value & P-value & Fold change \\
\hline 1 & E7ES66 & Glycocalicin & -9.835 & 0.0006 & 5.251 \\
\hline 2 & P02748 & Complement component C9 & 41.185 & $2.08 \mathrm{E}-06$ & 3.898 \\
\hline 3 & Q15848 & Adiponectin & 7.933 & 0.00137 & 3.575 \\
\hline 4 & P01833 & Polymeric immunoglobulin receptor & -3.682 & 0.02117 & 3.056 \\
\hline 5 & P10643 & Complement component C7 & 12.308 & 0.00025 & 2.900 \\
\hline 6 & F8W1Q3 & Biotinidase & -54.461 & $6.81 \mathrm{E}-07$ & 2.676 \\
\hline 7 & O95497 & Pantetheinase & -17.427 & $6.36 \mathrm{E}-05$ & 2.391 \\
\hline 8 & F6SYF8 & Dickkopf-related protein 2 & -19.531 & $4.05 \mathrm{E}-05$ & 2.178 \\
\hline 9 & P01024 & Complement C3 & 20.642 & $3.25 \mathrm{E}-05$ & 1.981 \\
\hline 10 & P04208 & Ig $\lambda$ chain V-I region WAH & -3.730 & 0.0203 & 1.918 \\
\hline 11 & P01019 & Angiotensinogen & -43.998 & $1.60 \mathrm{E}-06$ & 1.846 \\
\hline 12 & Q5VY30 & Plasma retinol-binding protein $(1-182)$ & -5.867 & 0.00421 & 1.830 \\
\hline 13 & F5H6IO & $\beta 2$-microglobulin & 30.320 & 7.05E-06 & 1.781 \\
\hline 14 & P02768 & Serum albumin & -51.884 & $8.26 \mathrm{E}-07$ & 1.723 \\
\hline 15 & P02760 & Protein AMBP & -11.934 & 0.00028 & 1.709 \\
\hline 16 & P10599 & Thioredoxin & 3.941 & 0.01694 & 1.647 \\
\hline 17 & P07355-2 & Annexin A2 & 26.293 & $1.24 \mathrm{E}-05$ & 1.622 \\
\hline 18 & P01857 & Ig $\gamma-1$ chain $C$ region & 6.790 & 0.00246 & 0.546 \\
\hline 19 & P04207 & Ig $\kappa$ chain V-III region CLL & 8.997 & 0.00084 & 0.534 \\
\hline 20 & P01861 & Ig $\gamma-4$ chain $C$ region & 8.329 & 0.00114 & 0.505 \\
\hline 21 & P02647 & Apolipoprotein A-I & 66.441 & $3.07 \mathrm{E}-07$ & 0.484 \\
\hline 22 & P58546 & Myotrophin & 4.805 & 0.00862 & 0.456 \\
\hline 23 & C9JIZ6 & Saposin-D & 7.474 & 0.00171 & 0.340 \\
\hline 24 & E9PGN7 & Plasma protease $\mathrm{C} 1$ inhibitor & 14.422 & 0.00013 & 0.327 \\
\hline 25 & P60174 & Triosephosphate isomerase & 15.263 & 0.00011 & 0.322 \\
\hline 26 & Q9H299 & $\begin{array}{l}\text { SH3 domain-binding glutamic acid- } \\
\text { rich-like protein } 3\end{array}$ & 8.893 & 0.00088 & 0.268 \\
\hline 27 & B7Z6Z4 & Myosin light polypeptide 6 & 24.641 & $1.61 \mathrm{E}-05$ & 0.151 \\
\hline 28 & Q14624-2 & $\begin{array}{l}\text { Isoform } 2 \text { of Inter- } \alpha \text {-trypsin inhibitor } \\
\text { heavy chain } \mathrm{H} 4\end{array}$ & 75.790 & $1.82 \mathrm{E}-07$ & 0.076 \\
\hline
\end{tabular}

GO annotation with respect to the 131 differentially expressed proteins in OA demonstrated that the cellular component of these proteins was predominantly derived from the extracellular matrix (ECM), cytoplasmic membrane, cytoplasm, secretory granule and lipoprotein particle. The molecular function of these proteins primarily included binding, transporter, enzyme activator and inhibitor activity. The biological process these proteins participated in predominantly included the acute inflammatory response, complement activation, immune response, response to stimulus, regulation of apoptosis and homeostatic process. KEGG pathway annotation indicated that these proteins were enriched in complement and coagulation cascades (Fig. 5), ECM-receptor interaction and the peroxisome proliferator activated receptor signaling pathway.

ELISA. As presented in Fig. 6, the mean level of complement C1r in SF from OA was 2,063.1 $\pm 1,238.1 \mathrm{ng} / \mathrm{ml}$, that from RA was $2,726.4 \pm 1,627.8 \mathrm{ng} / \mathrm{ml}$ and that from traumatic arthritis was $180.6 \pm 102.4 \mathrm{ng} / \mathrm{ml}$. The mean level of complement $\mathrm{C} 1 \mathrm{r}$ in SF from RA was significantly higher than those from OA
$(\mathrm{P}=0.033)$ and traumatic arthritis $(\mathrm{P}<0.001)$, and that from $\mathrm{OA}$ was also significantly higher than that from traumatic arthritis $(\mathrm{P}<0.001)$. According to the KL radiographic grading criteria, the mean level of complement C1r in SF from OA with KL grade 2 was $2,147.8 \pm 1,337.2 \mathrm{ng} / \mathrm{ml}$, and that from OA with KL grade 4 was $1,978.4 \pm 1,163.1 \mathrm{ng} / \mathrm{ml}$. The mean level of complement C1r in SF from traumatic arthritis was significantly lower than those from OA with KL grade $2(\mathrm{P}<0.001)$ and KL grade $4(\mathrm{P}<0.001)$; however, no significant difference was identified between the latter two $(\mathrm{P}=0.698)$. The levels of complement C1r in SF from OA were positively correlated with disease severity $(\mathrm{r}=0.549, \mathrm{P}<0.001)$. Similarly, the mean level of complement C1r in SF from RA with KL grade 2 was 2,920.9 $\pm 1,743.0 \mathrm{ng} / \mathrm{ml}$, and that from RA with KL grade 4 was $2,531.8 \pm 1,528.7 \mathrm{ng} / \mathrm{ml}$. In addition, the mean level of complement $\mathrm{C} 1 \mathrm{r}$ in SF from traumatic arthritis was significantly lower than those from RA with KL grade $2(\mathrm{P}<0.001)$ and KL grade $4(\mathrm{P}<0.001)$; however, no significant difference was identified between the latter two $(\mathrm{P}=0.374)$. The levels of complement $\mathrm{C} 1 \mathrm{r}$ in SF from RA were also positively correlated with disease severity $(r=0.540, \mathrm{P}<0.001)$. 

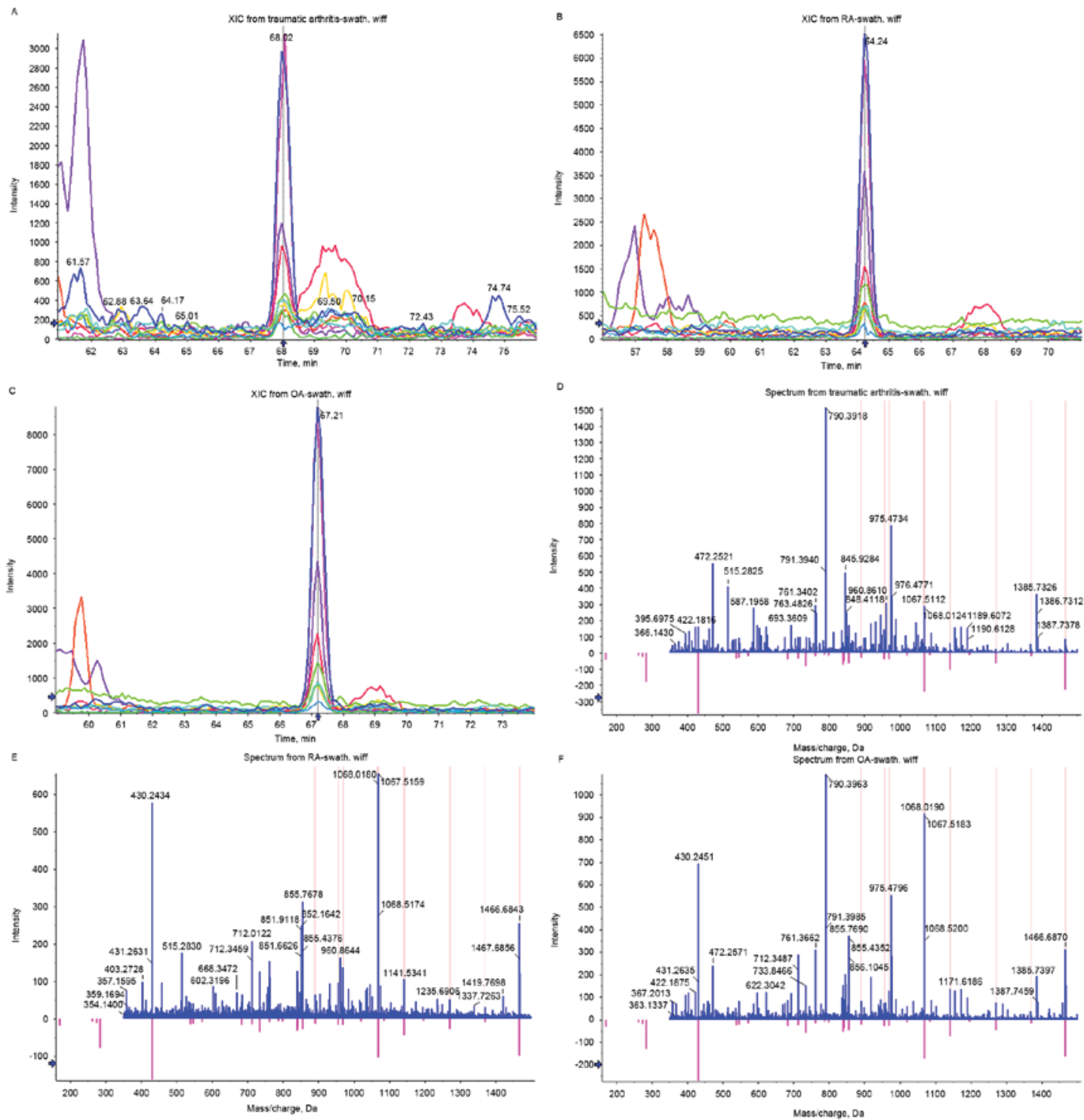

Figure 4. Quantification results of DKK2 using SWATH-MS.XICs indicated relative abundance of peptides from DKK2 in SF from (A) traumatic arthritis, (B) RA and (C) OA. Spectrum of peptides from DKK2 in SF from (D) traumatic arthritis, (E) RA and (F) OA. Relative quantification results derived from comparing the summed XIC peak areas of the fragment ion peak groups, as indicated by the arrow. DKK2, Dickkopf-related protein 2; SF, synovial fluid; MS, mass spectrometry; RA, rheumatoid arthritis; OA, osteoarthritis.

As shown Fig. 7, the mean level of DKK2 in SF from OA was $186.3 \pm 41.0 \mathrm{ng} / \mathrm{ml}$, that from RA was $93.0 \pm 34.5 \mathrm{ng} / \mathrm{ml}$ and that from traumatic arthritis was $74.2 \pm 22.9 \mathrm{ng} / \mathrm{ml}$. The mean level of DKK2 in SF from OA was significantly higher when compared with those from RA $(\mathrm{P}<0.001)$ and traumatic arthritis $(\mathrm{P}<0.001)$; however, no significant difference was identified between the latter two $(\mathrm{P}=0.069)$. According to the KL radiographic grading criteria, the mean level of DKK2 in $\mathrm{SF}$ from OA with KL grade 2 was $174.4 \pm 42.5 \mathrm{ng} / \mathrm{ml}$, and that from OA with KL grade 4 was $198.2 \pm 36.8 \mathrm{ng} / \mathrm{ml}$. The mean level of DKK2 in SF from OA with KL grade 4 was significantly higher than those from OA with KL grade $2(\mathrm{P}=0.045)$ and traumatic arthritis $(\mathrm{P}<0.001)$, and that from $\mathrm{OA}$ with $\mathrm{KL}$ grade 2 was also significantly higher than that from traumatic arthritis $(\mathrm{P}<0.001)$. The levels of DKK2 in SF from OA were positively correlated with disease severity $(r=0.796, P<0.001)$. Similarly, the mean level of DKK2 in SF from RA with KL grade 2 was $89.4 \pm 35.4 \mathrm{ng} / \mathrm{ml}$, and that from RA with $\mathrm{KL}$ grade 4 was $96.6 \pm 34.3 \mathrm{ng} / \mathrm{ml}$. No significant difference was detected between the mean levels of DKK2 in SF from RA with KL grade 2 and traumatic arthritis $(\mathrm{P}=0.195)$, between those from RA with KL grade 4 and traumatic arthritis $(\mathrm{P}=0.057)$ and between those from RA with KL grade 2 and KL grade $4(\mathrm{P}=0.537)$. In addition, the levels of DKK2 in SF from RA were positively correlated with disease severity $(r=0.287, \mathrm{P}=0.035)$.

\section{Discussion}

OA exerts a great influence on physical and psychological health of the elderly due to its high morbidity and disability rate (1). The lack of early diagnostic maneuvers and effective pharmacotherapy for OA is primarily attributed to the limitations in current understanding of its pathogenesis, which may be further elucidated by investigating the SF proteome. However, for a variety of reasons, previous studies with 
Table II. Differentially expressed proteins conversely regulated in OA and RA identified by SWATH-MS.

\begin{tabular}{lllllll}
\hline $\begin{array}{l}\text { Protein } \\
\text { ID }\end{array}$ & Accession & \multicolumn{1}{c}{ Protein name } & $\begin{array}{c}\text { P-value } \\
(\mathrm{OA})\end{array}$ & $\begin{array}{c}\text { Fold change } \\
(\mathrm{OA})\end{array}$ & $\begin{array}{c}\text { P-value } \\
(\mathrm{RA})\end{array}$ & $\begin{array}{c}\text { Fold change } \\
(\mathrm{RA})\end{array}$ \\
\hline 1 & P09211 & Glutathione S-transferase & 0.01097 & 2.051 & $2.87 \mathrm{E}-06$ & 0.527 \\
2 & P13611-2 & Isoform V1 of Versican core protein & $5.08 \mathrm{E}-05$ & 1.985 & $2.34 \mathrm{E}-05$ & 0.380 \\
3 & P10915 & Hyaluronan and proteoglycan link protein & $1.28 \mathrm{E}-05$ & 1.636 & $4.36 \mathrm{E}-06$ & 0.212 \\
4 & P37837 & Transaldolase & 0.00826 & 0.577 & 0.00022 & 5.286 \\
5 & P07737 & Profilin-1 & 0.03004 & 0.572 & $3.57 \mathrm{E}-05$ & 4.777 \\
6 & P63104 & 14-3-3 protein zeta/delta & $1.82 \mathrm{E}-07$ & 0.501 & $1.28 \mathrm{E}-05$ & 2.567 \\
7 & P06727 & Apolipoprotein A-IV & $1.73 \mathrm{E}-06$ & 0.463 & $1.24 \mathrm{E}-06$ & 1.536 \\
8 & P01880 & Ig delta chain C region & 0.02884 & 0.261 & $3.47 \mathrm{E}-05$ & 2.521 \\
\hline
\end{tabular}

OA, osteoarthritis; RA, rheumatoid arthritis.

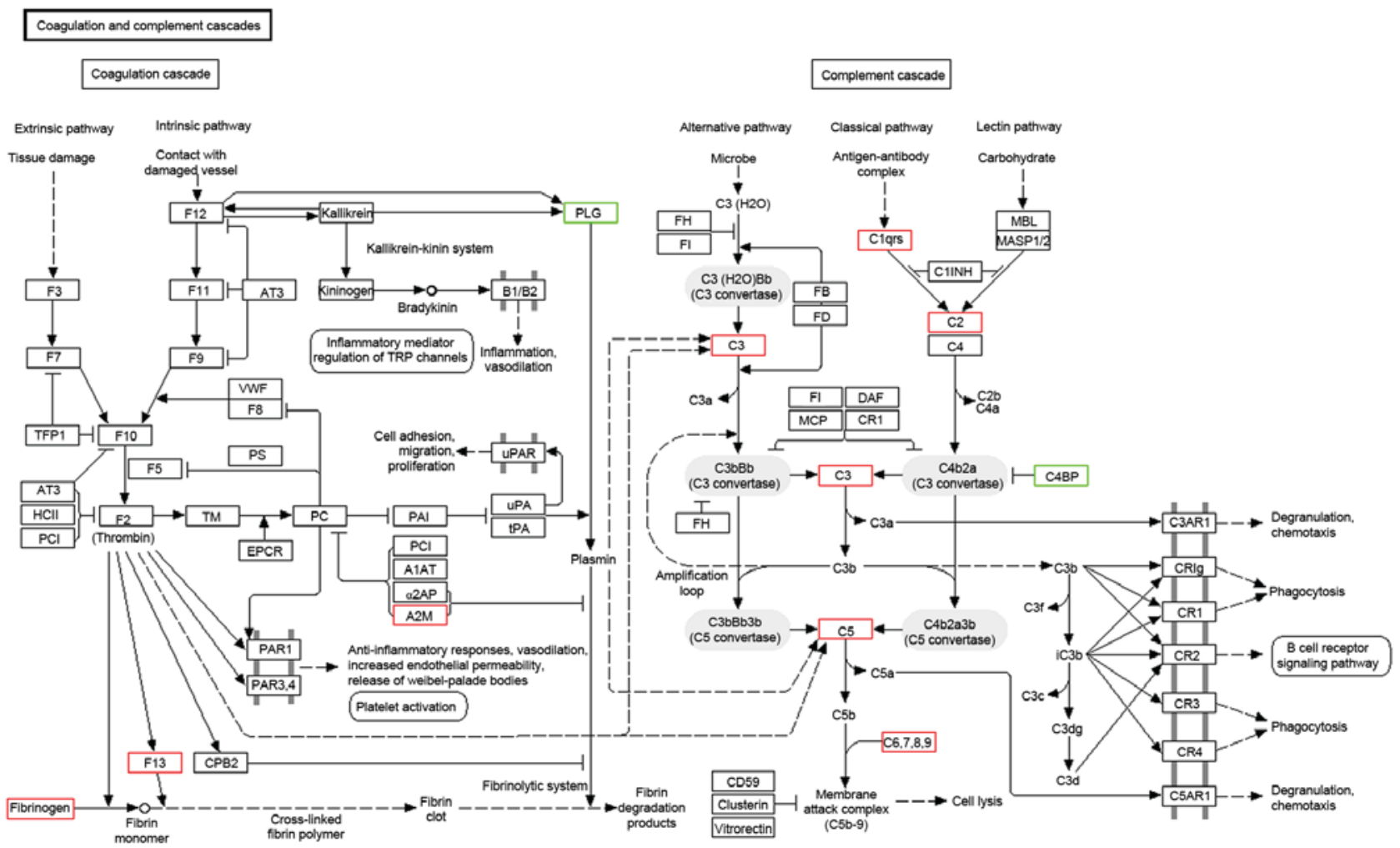

Figure 5. Pathway enrichment of differentially expressed proteins in complement and coagulation cascades. Proteins in red boxes corresponded to upregulation in OA and those in green boxes indicated downregulation in OA. OA, osteoarthritis.

regard to proteomic analysis of $\mathrm{OA}$ were not consistent in demonstrating the pathogenesis and failed to detect effective biomarkers or drug targets to facilitate early diagnosis and intervention of OA. Therefore, this provided the rationale for conducting the present study. In the current study, with the use of SWATH-MS, a total of 131 proteins were identified to be differentially expressed in SF from OA, of which 28 were specifically regulated in $\mathrm{OA}$ and eight were conversely regulated in $\mathrm{OA}$ and $\mathrm{RA}$. The quantity of differentially expressed proteins identified in the current study is markedly more than in previous studies analyzing SF proteome using other quantitative proteomic technologies $(7,8)$. With the use of two-dimensional polyacrylamide gel electrophoresis, Liu et al (15) identified 10 differential protein spots between the synovial membranes of the bilateral and unilateral knee OA in surgery-induced rabbit models. Similarly, Fernandez-Puente et al (16) developed a multiple reaction monitoring method with which a panel of 14 protein biomarker candidates for OA was identified. Differential proteins identified in the above-mentioned studies, such as lumican (15) and haptoglobin (16) were also identified in the current study. These indicate that, as a novel proteomic technology, SWATH-MS provides a more comprehensive quantification result in delineating theSF proteome and 

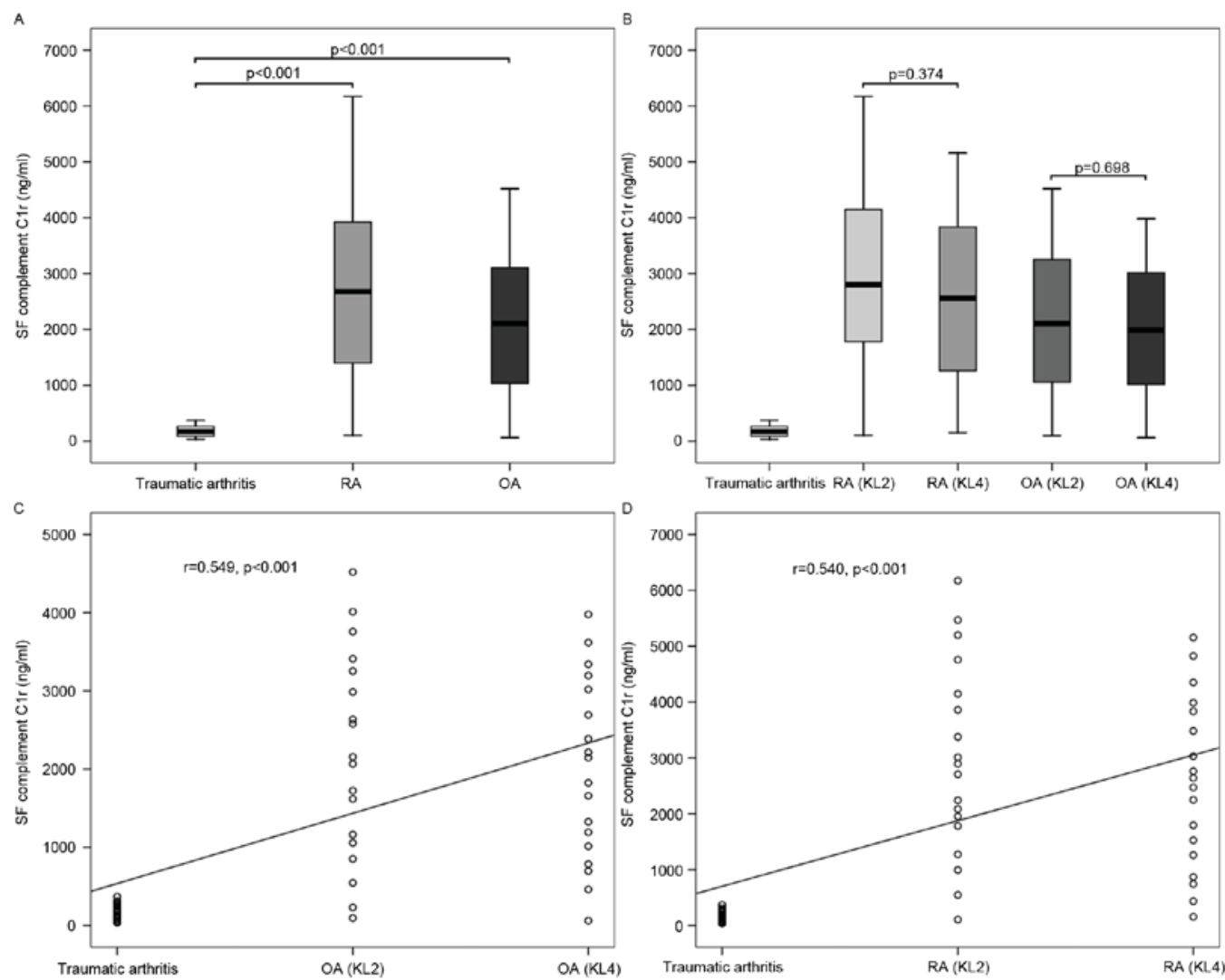

Figure 6. Enzyme-linked immunosorbent assay results of complement C1r. (A and B) Box plot presentation of complement C1r concentrations in synovial fluidfrom traumatic arthritis, RA and OA patients. (C and D) Scatter plot presentation of the associationbetween complement Clr concentrations and disease severity. The plot indicated that complement $\mathrm{C} 1 \mathrm{r}$ concentrations were positively correlated with $\mathrm{OA}(\mathrm{r}=0.549, \mathrm{P}<0.001)$ and $\mathrm{RA}$ severity $(\mathrm{r}=0.540, \mathrm{P}<0.001)$. RA, rheumatoid arthritis; OA, osteoarthritis; KL, Kellgren-Lawrence grade.
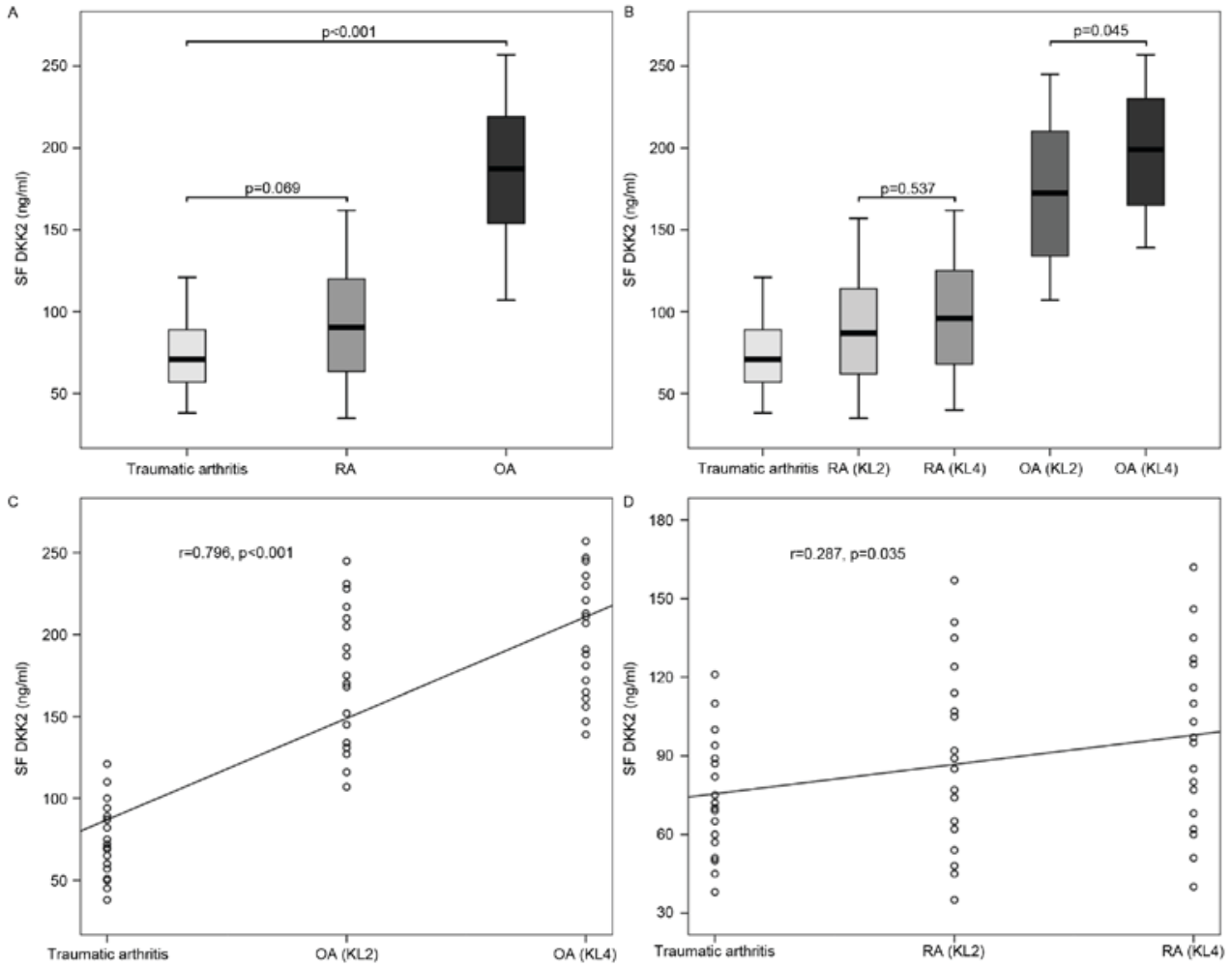

Figure 7. Enzyme-linked immunosorbent assay results of DKK2. (A and B) Box plot presentation of DKK2 concentrations in synovial fluidfrom traumatic arthritis, RA and OA patients. (C and D) Scatter plot presentation of the association between DKK2 concentrations and disease severity. The plot indicated that DKK2 concentrations were positively correlated with $\mathrm{OA}(\mathrm{r}=0.796, \mathrm{P}<0.001)$ and RA severity $(\mathrm{r}=0.287, \mathrm{P}=0.035)$. DKK2, Dickkopf-related protein 2; RA, rheumatoid arthritis; OA, osteoarthritis; KL, Kellgren-Lawrence grade. 
markedly benefits the screening of biomarkers due to its higher resolution and coverage rate.

The hypothesis that the complement system may participate in OA pathogenesis is substantiated by the identification of complement $\mathrm{C} 1 \mathrm{r}$ and enrichment of differentially expressed proteins in complement and coagulation cascades. The results are consistent with those reported by Wang et al (17), which indicates abnormally high expression levels and activation of complement components in SF and synovial membranes from OA. Complement activation in turn results in the formation of membrane attack complex (MAC) on chondrocytes, which either kills the cells or causes them to produce matrix-degrading enzymes, inflammatory mediators and other complement effectors, all of which promote joint pathology. A previous study indicates that MAC mediates collagenase expression and cartilage destruction primarily via extracellular signal-regulated kinase-dependent c-Fos induction (18). In addition, complement activation and MAC assembly also stimulate the cell cycle by inducing expression of c-Jun, JunD, and c-Fos mRNAs (19), which appears to have a potential correlation with the pathology of hyperosteogeny and synovial hyperplasia in OA. These findings provide a rationale for targeting the complement system as a disease-modifying therapeutic strategy for $\mathrm{OA}$.

DKK2 is one of the proteins that is specifically upregulated in OA, the alteration of which has been confirmed by subsequent ELISA in the present study. As a Wnt signaling antagonist, the overexpression of DKK2 contributes to the abnormal phenotype and poor mineralization of OA osteoblasts by altering the Wnt signaling pathway, which is crucial for osteogenesis and regulates terminal osteoblast differentiation (20). The upregulation of DKK2 in OA osteoblasts may be associated with the hypoxic condition and ischemic episode caused by modification of subchondral bone vascularization (21). Besides DKK2, there are many other proteins specifically regulated in OA. These proteins and their possible underlying mechanism in OA pathogenesis are as follows: i) Adiponectin is a protein hormone secreted predominantly by differentiated adipocytes, it increases intercellular adhesion molecule-1 (ICAM-1) expression levels in OA synovial fibroblasts via the liver kinase B1/calmodulin-dependent protein kinase II, AMP-activated protein kinase, c-Jun, and activator protein 1 signaling pathway. Adiponectin-induced ICAM-1 expression promotes the adhesion of monocytes to OA synovial fibroblasts, which may be involved in the pathogenesis of OA (22). Furthermore, adiponectin enhances production of nitric oxide, interleukin (IL)-6, matrix metalloproteinase (MMP)-1 and MMP-3 in OA chondrocytes in a mitogen-activated protein kinase-dependent manner (23). ii) The mechanisms by which $\beta 2$-microglobulin induces joint destruction is to induce the release of MMP-1 without concomitant release of tissue inhibitor of metalloproteinase-1 (TIMP-1) from OA synovial fibroblasts, leading to an increase in the MMP-1/TIMP-1 ratio, which is indicative of uncontrolled collagenolysis. The release of MMP-1 induced by $\beta 2$-microglobulin may be inhibited by doxycycline (24). iii) Thioredoxin, known as a cellular reducing catalyst induced by oxidative stress, is involved in the redox regulation of transcription factors, such as nuclear factor $(\mathrm{NF})-\kappa \mathrm{B}$. It appears to accelerate the nuclear translocation of $\mathrm{NF}-\kappa \mathrm{B}$, a major transcriptional regulator for production of
IL-6 and IL-8 on stimulation with tumor necrosis factor-a (25). iv) As for annexin A2, a discoidin domain receptor 2 (DDR-2) binding protein in the DDR-2/MMP signaling pathway, its phosphorylation by phosphorylated-DDR-2 leads to MMP-13 secretion (26).

Glutathione S-transferase (GST) is one of the proteins conversely regulated in OA and RA identified in the present study. The differential expression of GST in OA coincides with the results documented by Ostalowska et al (27), which indicate aberrant changes in the activities of antioxidant enzymes, including superoxide dismutase and GST in SF from OA. GST is critically important for cellular defense against oxidative stress-induced apoptosis in OA chondrocytes, possibly by elimination of 4-hydroxynonenal, one of the most abundant and reactive aldehydes of lipid peroxidation products (28). Hyaluronan and proteoglycan link protein is another protein conversely regulated in OA and RA. It has been reported that LP stabilizes the proteoglycan aggregates towards dissociation, and helps to protect them from degradation under conditions where tissue catabolism is promoted (29).

There were certain limitations of the current study. Due to ethical restrictions, patients undergoing arthroscopy for traumatic knee arthritis were selected, rather than healthy individuals, and served as the standard control group. However, as SF samples were acquired within 7 days after injury and patients in this group all exhibited intact articular cartilage under arthroscopy, it is reasonable for us to consider that these samples are most similar to those from healthy individuals in the proteomic profile. Furthermore, to investigate proteins specifically regulated in OA, the design of the inter-control group requires more diversification. In addition to RA, other arthropathies due to systemic lupus erythematosus, ankylosing spondylitis, gout, psoriasis or haemochromatosis should be included as inter-control groups.

In conclusion, the findings of the present study provide an improved understanding of the pathogenesis of OA, and may facilitate the early diagnosis and treatment of OA following the identification of various potential biomarkers and drug targets.

\section{Acknowledgements}

The authors would like to thank Dr Yang Liu for assistance in acquisition of SF samples, and Dr Can Huang for assistance with collection of patient data.

\section{References}

1. Neogi T: The epidemiology and impact of pain in osteoarthritis. Osteoarthritis Cartilage 21: 1145-1153, 2013.

2. Arnscheidt C, Meder A and Rolauffs B: Early diagnosis of osteoarthritis: Clinical reality and promising experimental techniques. Z Orthop Unfall 154: 254-268, 2016.

3. Jevsevar DS: Treatment of osteoarthritis of the knee: Evidence-based guideline, 2nd edition. J Am Acad Orthop Surg 21: 571-576, 2013.

4. Jiao Q, Wei L, Chen C, Li P, Wang X, Li Y, Guo L, Zhang C and Wei X: Cartilage oligomeric matrix protein and hyaluronic acid are sensitive serum biomarkers for early cartilage lesions in the knee joint. Biomarkers 21: 146-151, 2016.

5. Nemirovskiy O, Li WW and Szekely-Klepser G: Design and validation of an immunoaffinity LC-MS/MS assay for the quantification of a collagen type II neoepitope peptide in human urine: Application as a biomarker of osteoarthritis. Methods Mol Biol 641: 253-270, 2010. 
6. Duan Y, Hao D, Li M, Wu Z, Li D, Yang X and Qiu G: Increased synovial fluid visfatin is positively linked to cartilage degradation biomarkers in osteoarthritis. Rheumatol Int 32: 985-990, 2012.

7. Yamagiwa H, Sarkar G, Charlesworth MC, McCormick DJ and Bolander ME: Two-dimensional gel electrophoresis of synovial fluid: Method for detecting candidate protein markers for osteoarthritis. J Orthop Sci 8: 482-490, 2003.

8. Gobezie R, Kho A, Krastins B, Sarracino DA, Thornhill TS, Chase M, Millett PJ and Lee DM: High abundance synovial fluid proteome: Distinct profiles in health and osteoarthritis. Arthritis Res Ther 9: R36, 2007.

9. Wu WW, Wang G, Baek SJ and Shen RF: Comparative study of three proteomic quantitative methods, DIGE, cICAT and iTRAQ, using 2D gel- or LC-MALDI TOF/TOF. J Proteome Res 5: 651-658, 2006.

10. Balakrishnan L, Bhattacharjee M, Ahmad S, Nirujogi RS, Renuse S, Subbannayya Y, Marimuthu A, Srikanth SM, Raju R and Dhillon M: Differential proteomic analysis of synovial fluid from rheumatoid arthritis and osteoarthritis patients. Clin Proteomics 11: 1, 2014.

11. Lourido L, Calamia V, Mateos J, Fernández-Puente P, Fernández-Tajes J, Blanco FJ and Ruiz-Romero C: Quantitative proteomic profiling of human articular cartilage degradation in osteoarthritis. J Proteome Res 13: 6096-6106, 2014

12. Gillet LC, Navarro P, Tate S, Röst H, Selevsek N, Reiter L, Bonner R and Aebersold R: Targeted data extraction of the MS/MS spectra generated by data-independent acquisition: A new concept for consistent and accurate proteome analysis. Mol Cell Proteomics 11: O111.016717, 2012.

13. Collins BC, Gillet LC, Rosenberger G, Röst HL, Vichalkovski A, Gstaiger M and Aebersold R: Quantifying protein interaction dynamics by SWATH mass spectrometry: Application to the 14-3-3 system. Nat Methods 10: 1246-1253, 2013.

14. Aletaha D, Neogi T, Silman AJ, Funovits J, Felson DT, Bingham CO III, Birnbaum NS, Burmester GR, Bykerk VP and Cohen MD: 2010 Rheumatoid arthritis classification criteria: An american college of rheumatology/european league against rheumatism collaborative initiative. Arthritis Rheum 62: 2569-2581, 2010.

15. Liu W, He J, Lin R, Liang J and Luo Q: Differential proteomics of the synovial membrane between bilateral and unilateral knee osteoarthritis in surgery-induced rabbit models. Mol Med Rep 14: 2243-2249, 2016.

16. Fernandez-Puente P, Calamia V, González-Rodríguez L, Lourido L, Camacho-Encina M, Oreiro N, Ruiz-Romero C and Blanco FJ: Multiplexed mass spectrometry monitoring of biomarker candidates for osteoarthritis. J Proteomics 152: 216-225, 2017.

17. Wang Q, Rozelle AL, Lepus CM, Scanzello CR, Song JJ, Larsen DM, Crish JF, Bebek G, Ritter SY and Lindstrom TM: Identification of a central role for complement in osteoarthritis. Nat Med 17: 1674-1679, 2011.

18. Litherland GJ, Elias MS, Hui W, Macdonald CD, Catterall JB, Barter MJ, Farren MJ, Jefferson M and Rowan AD: Protein kinase $\mathrm{C}$ isoforms zeta and iota mediate collagenase expression and cartilage destruction via STAT3- and ERK-dependent c-fos induction. J Biol Chem 285: 22414-22425, 2010.
19. Rus HG, Niculescu F and Shin ML: Sublytic complement attack induces cell cycle in oligodendrocytes. J Immunol 156: 4892-4900, 1996.

20. Chan TF, Couchourel D, Abed E, Delalandre A, Duval N and Lajeunesse D: Elevated Dickkopf-2 levels contribute to the abnormal phenotype of human osteoarthritic osteoblasts. J Bone Miner Res 26: 1399-1410, 2011.

21. Bouvard B, Abed E, Yéléhé-Okouma M, Bianchi A Mainard D, Netter P, Jouzeau JY, Lajeunesse D and Reboul P: Hypoxia and vitamin D differently contribute to leptin and dickkopf-related protein 2 production in human osteoarthritic subchondral bone osteoblasts. Arthritis Res Ther 16: 459, 2014.

22. Chen HT, Tsou HK, Chen JC, Shih JM, Chen YJ and Tang CH: Adiponectin enhances intercellular adhesion molecule-1 expression and promotes monocyte adhesion in human synovial fibroblasts. PLoS One 9: e92741, 2014

23. Koskinen A, Juslin S, Nieminen R, Moilanen T, Vuolteenaho K and Moilanen E: Adiponectin associates with markers of cartilage degradation in osteoarthritis and induces production of proinflammatory and catabolic factors through mitogen-activated protein kinase pathways. Arthritis Res Ther 13: R184, 2011.

24. Moe SM, Singh GK and Bailey AM: beta2-microglobulin induces MMP-1 but not TIMP-1 expression in human synovial fibroblasts. Kidney Int 57: 2023-2034, 2000.

25. Yoshida S, Katoh T, Tetsuka T, Uno K, Matsui N and Okamoto T: Involvement of thioredoxin in rheumatoid arthritis: Its costimulatory roles in the TNF-alpha-induced production of IL-6 and IL-8 from cultured synovial fibroblasts. J Immunol 163: 351-358, 1999.

26. Zhao W, Zhang C, Shi M, Zhang J, Li M, Xue X, Zhang Z, Shu Z, Zhu $\mathrm{J}$ and $\mathrm{Mu} \mathrm{N}$ : The discoidin domain receptor $2 /$ annexin A2/matrix metalloproteinase 13 loop promotes joint destruction in arthritis through promoting migration and invasion of fibroblast-like synoviocytes. Arthritis Rheumatol 66: 2355-2367, 2014.

27. Ostalowska A, Birkner E, Wiecha M, Kasperczyk S, Kasperczyk A, Kapolka D and Zon-Giebel A: Lipid peroxidation and antioxidant enzymes in synovial fluid of patients with primary and secondary osteoarthritis of the knee joint. Osteoarthritis Cartilage 14: 139-145, 2006.

28. Vaillancourt F, Fahmi H, Shi Q, Lavigne P, Ranger P, Fernandes JC and Benderdour M: 4-Hydroxynonenal induces apoptosis in human osteoarthritic chondrocytes: The protective role of glutathione-S-transferase. Arthritis Res Ther 10: R107, 2008.

29. Rodriguez E and Roughley P: Link protein can retard the degradation of hyaluronan in proteoglycan aggregates. Osteoarthritis Cartilage 14: 823-829, 2006.

This work is licensed under a Creative Commons Attribution-NonCommercial-NoDerivatives 4.0 International (CC BY-NC-ND 4.0) License. 